ITFA-2007-49

\title{
BRST quantization of the pure spinor superstring
}

\author{
Joost Hoogeveen and Kostas Skenderis \\ Institute for Theoretical Physics, University of Amsterdam, \\ Valckenierstraat 65, 1018XE Amsterdam, The Netherlands \\ joosth, skenderi@science.uva.nl
}

\begin{abstract}
$\underline{\text { ABSTRACT }}$
We present a derivation of the scattering amplitude prescription for the pure spinor superstring from first principles, both in the minimal and non-minimal formulations, and show that they are equivalent. This is achieved by first coupling the worldsheet action to topological gravity and then proceeding to BRST quantize this system. Our analysis includes the introduction of constant ghosts and associated auxiliary fields needed to gauge fix symmetries associated with zero modes. All fields introduced in the process of quantization can be integrated out explicitly, resulting in the prescriptions for computing scattering amplitudes that have appeared previously in the literature. The zero mode insertions in the path integral follow from the integration over the constant auxiliary fields.
\end{abstract}




\section{Contents}

1 Introduction

2 Review of the pure spinor formalism 4

3 Coupling to 2d gravity 6

3.1 Topological gravity and $Q_{S}$ invariance . . . . . . . . . . . . . . 9

4 Adding vertex operators $\quad 10$

5 BRST quantization $\quad 11$

5.1 Summary ............................ 14

6 Pure spinor measure $\quad 15$

6.1 Minimal formulation . . . . . . . . . . . . . . . 18

6.2 Non-minimal formulation . . . . . . . . . . . . . . . 20

$\begin{array}{lll}7 & \text { Conclusions } & 21\end{array}$

A $U(5)$ variables and the $Y$-formalism $\quad 23$

B Ghost contribution $\quad 26$

\section{Introduction}

The covariant super-Poincaré quantization of the ten dimensional superstring is an important problem that has attracted lots of attention over the years. There are strong motivations for developing such a formalism. To start with one would like to be able to compute multi-loop amplitudes in a manifestly supersymmetric fashion and analyze the associated issue of the perturbative finiteness of the superstring perturbation theory. Furthermore, holographic dualities and the study of flux vacua in string theory make urgent the need for a formalism that can handle RR backgrounds.

A new formalism that achieves such a covariant quantization, the pure spinor formalism, was proposed by Berkovits in [1], see [2] for a review. The worldsheet fields, in the minimal version of this formalism, are the spacetime coordinates $X^{m}$ and 
the spacetime fermions $\theta^{\alpha}$, as in the Green-Schwarz formalism, conjugate fermionic momenta $p_{\alpha}$ (first introduced by Siegel in [3]) and new bosonic twistor-like variables $\lambda^{\alpha}$ that take values in the space of pure spinors, namely they satisfy $\lambda^{\alpha} \gamma_{\alpha \beta}^{m} \lambda^{\beta}=0$, and their conjugate momenta $w_{\alpha}$. The non-minimal version contains an additional bosonic pure spinor of opposite chirality, $\bar{\lambda}_{\alpha}$, a constrained fermionic spinor $r_{\alpha}$ and their conjugate momenta, $\bar{w}^{\alpha}, s^{\alpha}$. One can construct from these fields a fermionic nilpotent operator $Q_{S}$ that is postulated to be the BRST operator of the theory. In a flat background the worldsheet theory is free (modulo the non-linear pure spinor constraint) and a prescription for the computation of scattering amplitudes has been developed in a number of papers [1, 4, 5, 6] with tests and explicit computations presented in [7, 8, 9, 10, 11].

There are several unconventional aspects of this formalism. Usually the BRST symmetry arises after gauge fixing a local symmetry, which in the case of strings includes worldsheet diffeomorphisms. In the pure spinor formalism however one instead is given directly the "gauged-fixed" model in the conformal gauge and a BRST operator $Q_{S}$. Similarly, the prescription for the scattering amplitudes was postulated rather than derived from first principles. In particular, the absence of an antighost field $b$ led to a (complicated) construction of a composite field, with properties similar to that of the antighost, which was used in the proposal for the measure of the multi-loop amplitudes. Although there is very little doubt that the current form of the computation rules is correct, it would clearly be desirable to have a first principles derivation. Such a derivation, apart from providing a better justification of the current computational rules, could also help in the search of a simplified version.

In this paper we provide such a derivation. There have been many works in the past involving modifications and/or extensions of the pure spinor formalism with the same aim, see for example [12, 13, 14, 15, 16, 17, 18, 19]. Our approach is different and is guided by topological string constructions. Instead of searching for a model with a local symmetry which after gauge fixing would lead to the pure spinor formalism with $Q_{S}$ and the pure spinors emerging as a BRST operator and ghost fields, we shall consider the pure spinors $\lambda$ as "matter" fields as well and the worldsheet theory as a sigma model with a nilpotent symmetry $Q_{S}$ and target space ten dimensional superspace times the pure spinor space. To construct a string theory we couple this 
theory to two-dimensional gravity in a way that preserves the fermionic symmetry $Q_{S}$ and then BRST quantize the resulting theory in a conventional fashion. Following [20], gauge invariances due to zero modes are also included in the BRST analysis by introducing constant ghosts. This leads automatically to a scattering amplitude prescription that is BRST invariant and upon integrating out the constant ghosts and associated auxiliary fields one arrives at various insertions in the path integral measure.

This paper is organized as follows. In the next section we review the pure spinor formalism. In section 3 we couple the theory to $2 d$ topological gravity and in section 4 we introduce vertex operators. The BRST quantization of this system is presented in section 5. Section 6 contains the analysis of the invariances due to the pure spinor zero modes. In this section we show that depending on how one treats the auxiliary fields one arrives either at the minimal or the non-minimal scattering amplitude prescription. We conclude in section 7 with a brief summary of our results. Finally there are two appendices: in the first we discuss $U(5)$ variables and the $Y$ formalism, while the second contains details of computations relevant for section 5 .

\section{Review of the pure spinor formalism}

We review in this section the pure spinor formalism. In the minimal version, the worldsheet action for the left-movers in conformal gauge and flat target space is given by

$$
S_{\sigma}=\int d^{2} z\left(\frac{1}{2} \partial x^{m} \bar{\partial} x_{m}+p_{\alpha} \bar{\partial} \theta^{\alpha}-w_{\alpha} \bar{\partial} \lambda^{\alpha}\right)
$$

with $m=0, \ldots, 9$ and $\alpha=1, \ldots, 16$. For Type II strings the right-movers are similar to the left-movers while for the heterotic string the right-moving variables are those of the heterotic RNS formalism. The field $\lambda^{\alpha}$ is a bosonic pure spinor satisfying,

$$
\lambda^{\alpha} \gamma_{\alpha \beta}^{m} \lambda^{\beta}=0
$$

where $\gamma_{\alpha \beta}^{m}$ are the symmetric $16 \times 16 d=10$ Pauli matrices. The fields $\theta^{\alpha}, \lambda^{\alpha}$, have conformal dimension 0 and the corresponding conjugate momenta $p_{\alpha}, w^{\alpha}$ have conformal dimension 1.

Since the action is quadratic in fields quantization is straightforward except for the fact that $\lambda^{\alpha}$ satisfies the quadratic constraint (2.2), so its quantization requires some 
explanation. More precisely, the pure spinor part of the action is a curved $\beta \gamma$ system describing maps from the worldsheet to the space of pure spinors, with $\lambda^{\alpha}$ being holomorphic coordinates in this space. This system can be analyzed by covering the space of pure spinors with coordinate patches and then gluing appropriately on the overlaps [21, 22]. In particular, one may cover the pure spinor space with 16 coordinate patches chosen such that on each of them one of the pure spinor components $\lambda^{\alpha}$ is non-vanishing. On such a patch one may explicitly solve the pure spinor constraint to express $\lambda^{\alpha}$ in terms of 11 independent (complex) variables. For example, using the decomposition $\underline{16} \rightarrow \underline{1}+\underline{10}+\underline{5}^{*}$ of the spinor of (the Wick rotated Lorentz group) $S O(10)$ under $S U(5)$ one may solve the pure spinor constraint by suitably expressing the $\underline{5}^{*}$ in terms of the $\underline{1}$ and $\underline{10}$. Furthermore, the action (2.1) has a gauge invariance

$$
\delta w_{\alpha}=\Lambda_{m}\left(\gamma^{m} \lambda\right)_{\alpha}
$$

where $\Lambda^{m}$ is a gauge parameter, which on each patch can be used to eliminate 5 components of $w_{\alpha}$, so we are left with 11 conjugate momenta for the 11 independent pure spinor components. In appendix $\mathrm{A}$ we show how to implement these steps in the path integral starting from a Lorentz invariant action involving unconstrained spinors $\lambda^{\alpha}$ and a Lagrange multiplier $l_{m}$ that imposes the pure spinor condition. Integrating out the Lagrange multiplier and the ghost fields resulting from gauge fixing the invariance (2.3) one obtains (after a non-trivial cancellation) a free action for the 11 independent pure spinor variables and their conjugate momenta. Since the starting point is Lorentz invariant all computations done with the $U(5)$ variables will preserve Lorentz invariance.

The model is invariant under a fermionic nilpotent symmetry (which for the leftmovers is) generated by

$$
Q_{S}=\oint d z \lambda^{\alpha}(z) d_{\alpha}(z)
$$

where

$$
d_{\alpha}=p_{\alpha}-\frac{1}{2} \gamma_{\alpha \beta}^{m} \theta^{\beta} \partial x_{m}-\frac{1}{8} \gamma_{\alpha \beta}^{m} \gamma_{m} \gamma \delta \theta^{\beta} \theta^{\gamma} \partial \theta^{\delta},
$$

which is considered to play the role of the BRST operator. The transformations it generates are given by

$$
\delta_{S} x^{m}=\lambda \gamma^{m} \theta, \quad \delta_{S} \theta^{\alpha}=\lambda^{\alpha}, \quad \delta_{S} \lambda^{\alpha}=0, \quad \delta_{S} d_{\alpha}=-\Pi^{m}\left(\gamma_{m} \lambda\right)_{\alpha}, \quad \delta_{S} w_{\alpha}=d_{\alpha}
$$


where $\Pi^{m}=\partial x^{m}+\frac{1}{2} \theta \gamma^{m} \partial \theta$ is the supersymmetric momentum and again we restrict to the left-movers (so in particular, the full transformation for $x^{m}$ contains a similar additive term with right-moving fields). The cohomology of this operator (at ghost number one) indeed correctly reproduces the superstring spectrum [23].

The non-minimal version of the formalism [5] amounts to introducing a set of nonminimal variables, the complex conjugate $\bar{\lambda}_{\alpha}$ of $\lambda^{\alpha}$, a fermionic constrained spinor $r_{\beta}$ satisfying

$$
\bar{\lambda}_{\alpha} \gamma_{m}^{\alpha \beta} \bar{\lambda}_{\beta}=0, \quad \bar{\lambda}_{\alpha} \gamma_{m}^{\alpha \beta} r_{\beta}=0
$$

and their conjugate momenta, $\bar{w}^{\alpha}$ and $s^{a}$. The action (2.1) is modified by the addition of the term $S_{n m}$

$$
S_{\sigma} \rightarrow S_{\sigma}+S_{n m}, \quad S_{n m}=\int d^{2} z\left(-\bar{w}^{\alpha} \bar{\partial} \bar{\lambda}_{\alpha}+s^{\alpha} \bar{\partial} r_{\alpha}\right)
$$

and the generator $Q_{S}$ by

$$
Q_{S} \rightarrow Q_{S}+\oint d z \bar{w}^{\alpha} r_{\alpha}
$$

This acts on the non-minimal variables as follows

$$
\delta_{S} \bar{\lambda}_{\alpha}=r_{\alpha}, \quad \delta_{S} r_{\alpha}=0, \quad \delta_{S} s^{\alpha}=\bar{w}^{\alpha}, \quad \delta_{S} \bar{w}^{\alpha}=0
$$

These transformation rules imply that the cohomology is independent of the nonminimal variables.

\section{Coupling to 2d gravity}

To construct a string theory we will couple the theory discussed in the previous section to two-dimensional gravity in a way that preserves the $Q_{S}$ symmetry and then quantize this system. Since this model has zero central charge, one should couple it to topological gravity. Our approach is thus similar to the construction of topological string theories, see [24] for a review. In that context one starts from a supersymmetric sigma model which upon topological twisting yields a topological sigma model. In this procedure one of the supersymmetry charges is identified with the BRST operator of the sigma model. The corresponding operator in our case is the nilpotent operator $Q_{S}$. Note that the pure spinor sigma model has been obtained by twisting an $N=2$ model in [16]. 
The first step in this procedure is thus to relax the conformal gauge in the action (2.1) (or (2.8) for the non-minimal version). The part that involves the $x^{m}$ is standard 1 ,

$$
S_{X}=\int d^{2} \sigma\left(\frac{1}{4} \sqrt{g} g^{a b} \partial_{a} x^{m} \partial_{b} x_{m}\right)
$$

The rest of the action (2.1) (or (2.8) for the non-minimal version) is a sum of first order actions involving a field of dimension one and a field of dimension zero (with an overall sign that depends on whether the fields are bosonic or fermionic). The covariantization of all these terms is the same, so it suffices to discuss one of them, say

$$
S_{(p, \theta)}=\int d^{2} z p_{\alpha} \bar{\partial} \theta^{\alpha}
$$

The fields of dimension one are vectors on the worldsheet, so $p_{\alpha}$ is more accurately labeled as $p_{a \alpha}$. However, only the $z$-component participates in (3.2). Similarly, only the $\bar{z}$ component of the right-moving momentum $2 \tilde{p}_{a \alpha}$ participates in the action. To account for this, we introduce the projection operators

$$
P_{a}^{( \pm) b}=\frac{1}{2}\left(\delta_{a}^{b} \mp i J_{a}^{b}\right)
$$

where $J_{a}{ }^{b}$ is the complex structure of the worldsheet, i.e. it satisfies

$$
J_{a}{ }^{b} J_{b}{ }^{c}=-\delta_{a}^{c}, \quad \nabla_{c} J_{a}{ }^{b}=0 .
$$

In terms of the worldsheet volume form and the worldsheet metric, it is given by $J_{a}{ }^{b}=-\epsilon_{a c} g^{c b}$, with $\epsilon_{a b}=\sqrt{g} \hat{\epsilon}_{a b}$ and $\hat{\epsilon}_{01}=1$, and holomorphic and anti-holomorphic functions on the worldsheet are defined by $J_{a}{ }^{b} \partial_{b} f=i \partial_{a} f$ and $J_{a}{ }^{b} \partial_{b} \tilde{f}=-i \partial_{a} \tilde{f}$, respectively. Using (3.4) one shows that

$$
P_{a}^{( \pm) b} P_{b}^{( \pm) c}=P_{a}^{( \pm) c} \quad P_{a}^{( \pm) b} P_{b}^{(\mp) c}=0 .
$$

Notice also that

$$
g^{a b} P_{b}^{( \pm) c}=g^{c b} P_{b}^{(\mp) a} .
$$

\footnotetext{
${ }^{1}$ We work with an Euclidean worksheet and use standard conventions, i.e. $z=\sigma^{1}+i \sigma^{2}$, the flat metric is $g_{z \bar{z}}=1 / 2$ etc.

${ }^{2}$ Note that throughout this article we use the notation that right-moving fields have a tilde (rather than the more conventional bar).
} 
One can obtain vectors with only $z$-component by multiplying by $P_{a}^{(+) b}$ and vectors with only $\bar{z}$-component by multiplying by $P_{a}^{(-) b}$ :

$$
\hat{p}_{a}=P_{a}^{(+) b} p_{b}, \quad \hat{\tilde{p}}_{a}=P_{a}^{(-) b} \tilde{p}_{b}
$$

In other words, in complex coordinates the only non-zero component of $P_{a}^{(+) b}$ is $P_{z}^{(+) z}=1$ and the only non-zero component of $P_{a}^{(-) b}$ is $P_{\bar{z}}^{(-) \bar{z}}=1$. More generally, these projection operators can be used to covariantize any tensor given in conformal gauge. The action (3.2) can then be covariantized as

$$
S_{(p, \theta)}=\int d^{2} \sigma \sqrt{g} g^{a b} \hat{p}_{a \alpha} \partial_{b} \theta^{\alpha}
$$

In summary the action of the minimal model coupled to gravity is given by

$$
S_{\sigma}=\int d^{2} \sigma \sqrt{g} g^{a b}\left(\frac{1}{4} \partial_{a} x^{m} \partial_{b} x_{m}+\hat{p}_{a \alpha} \partial_{b} \theta^{\alpha}-\hat{w}_{a \alpha} \partial_{b} \lambda^{\alpha}\right)
$$

with an obvious addition for the case of the non-minimal model. The stress energy tensor for the model can be obtained by varying w.r.t. the worldsheet metric,

$$
\begin{aligned}
T_{a b} & =\frac{2}{\sqrt{g}} \frac{\delta S_{\sigma}}{\delta g^{a b}}=\frac{1}{2}\left(\partial_{a} x_{m} \partial_{b} x^{m}-\frac{1}{2} g_{a b} g^{c d} \partial_{c} x_{m} \partial_{d} x^{m}\right) \\
& +\left(p_{(a|\alpha|} \partial_{b)} \theta^{\alpha}-\frac{1}{2} g_{a b} g^{c d} p_{c \alpha} \partial_{d} \theta^{\alpha}\right)+T_{a b}^{(\lambda w)}
\end{aligned}
$$

The contribution of the pure spinor part (and the non-minimal variables) is same as the one for the $(p, \theta)$ part with $p \rightarrow w$ and $\theta \rightarrow \lambda$ and an overall minus sign (with similar replacements for the non-minimal fields). This stress energy tensor is (manifestly) traceless and covariantly conserved, reflecting the fact that the action is invariant under diffeomorphisms and Weyl transformations,

$$
\begin{aligned}
\delta g_{a b} & =\mathcal{L}_{\epsilon(\sigma)} g_{a b}+2 \phi(\sigma) g_{a b} \\
\delta \Phi & =-\epsilon^{a} \partial_{a} \Phi \\
\delta P_{a} & =-\epsilon^{a} \partial_{a} P+\partial_{a} \epsilon^{b} P_{b}
\end{aligned}
$$

where $\epsilon^{a}(\sigma), \phi(\sigma)$ are diffeomorphism and Weyl gauge parameters, $\mathcal{L}_{\epsilon}$ is the Lie derivative, $\Phi=\left\{x^{m}, \theta^{\alpha}, \lambda^{\alpha}, \ldots\right\}$ denotes collectively all worldsheet scalars and $P_{a}=$ $\left\{p_{a \alpha}, w_{a \alpha}, \ldots\right\}$ denotes collectively all worldsheet vectors.

The stress energy tensor (3.10) can be rewritten as

$$
T_{a b}=P_{a}^{(+) c} P_{b}^{(+) d} T_{c d}^{B}+P_{a}^{(-) c} p_{c \alpha}\left(P_{b}^{(-) d} \partial_{d} \theta^{\alpha}\right)+\cdots
$$


where the dots indicate the contribution from the pure spinor and non-minimal variables, which will be suppressed from now on since they are similar to the $(p, \theta)$ contribution. We also suppress the anti-holomorphic contribution of $x^{m}$. The first term in (3.12) is the covariantization of the stress energy tensor appearing in Berkovits' work,

$$
T_{a b}^{B}=\frac{1}{2} \partial_{a} x_{m} \partial_{b} x^{m}+p_{a \alpha} \partial_{b} \theta^{\alpha}+\cdots
$$

while the second term is proportional to the $\theta^{\alpha}$ field equation. This additional term can be removed by modifying the transformation rule of $p_{a \alpha}$ in (3.11).

\subsection{Topological gravity and $Q_{S}$ invariance}

If we were to quantize the model just described we would find that it is anomalous, since the diffeomorphism ghosts would contribute $c=-26$ and the original sigma model had $c=0$. This problem is avoided by extending the $Q_{S}$ symmetry to act on the worldsheet metric, so that the $2 d$ gravity is topological. With this aim, we introduce the following transformation rule,

$$
\delta_{S} g_{a b}=P_{a}^{(-) c} P_{b}^{(-) d} \psi_{c d} \equiv \hat{\psi}_{a b}, \quad \delta_{S} \hat{\psi}_{c d}=0
$$

where $\psi_{a b}$ is a new field that has only one holomorphic component, $\psi_{\bar{z} \bar{z}}(z)$. (To extend this discussion to the anti-holomorphic sector we would need to also turn on $\tilde{\psi}_{z z}(\bar{z})$, i.e. the full transformation is $\left.\delta_{S} g_{a b}=P_{a}^{(-) c} P_{b}^{(-) d} \psi_{c d}+P_{a}^{(+) c} P_{b}^{(+) d} \tilde{\psi}_{c d}\right)$.

Since the metric now transforms, the action is not invariant and its $Q_{S}$ variation yields,

$$
\delta_{S} S_{\sigma}=-\frac{1}{2} \int d^{2} \sigma \sqrt{g} T^{a b} \delta_{S} g_{a b}=-\frac{1}{2} \int d^{2} \sigma \sqrt{g} g^{a c} g^{b d} T_{a b}^{B} \hat{\psi}_{c d}
$$

where again we only discuss the holomorphic sector, and in the second equality we used the fact that due to the projector operators the second term in (3.12) does not contribute. To construct an invariant action we now add a new term to the action,

$$
S_{\sigma} \rightarrow S=S_{\sigma}+\frac{1}{2} \int d^{2} \sigma \sqrt{g} g^{a c} g^{b d} G_{a b} \hat{\psi}_{c d}
$$

The new action would be invariant provided there exists $G_{a b}$ transforming as

$$
\delta_{S} G_{a b}=T_{a b}^{B}
$$


Note that because $\hat{\psi}_{a b}$ has only one fermionic component, the variation of the explicit worldsheet metrics in the new term does not contribute. Including both sectors one finds that for the discussion to go through $G_{a b}$ must be traceless. Equation (3.17) for $G_{a b}$ is precisely the equation for a composite "b-field". Such a composite field has been constructed in conformal gauge and one may covariantize it to obtain a $Q_{S}$, diffeomorphism and Weyl invariant action. We will come back to the solution of (3.17) later on.

\section{Adding vertex operators}

We will be interested in computing scattering amplitudes. For this aim, it is useful to introduce sources $\rho^{i}$ with Weyl weight one that couple to vertex operators $V_{i}$ that are scalar functionals with Weyl weight minus one [20]. Then our starting point is the extended action

$$
\mathcal{S}=S+\sum_{i=1}^{n} \rho^{i} V_{i}[\varphi]\left(\sigma_{i}, \zeta_{i}\right)
$$

where $S$ is given in (3.16),$\varphi$ denotes collectively all worldsheet fields and we will shortly discuss the vertex operator. The sources $\rho^{i}$ are considered to be infinitesimal, i.e. we only differentiate once with respect to each source and then set them to zero. The new action (4.1) depends on the positions of the vertex operators $\sigma_{i}^{a}$ and their $Q_{S}$ partners $\zeta_{i}^{a}$,

$$
\delta_{S} \sigma_{i}^{a}=\zeta_{i}^{a}, \quad \delta_{S} \zeta_{i}^{a}=0
$$

or in complex coordinates,

$$
\delta_{S} z_{i}=\zeta_{i}, \quad \delta_{S} \bar{z}_{i}=\bar{\zeta}_{i}, \quad \delta_{S} \zeta_{i}=0, \quad \delta_{S} \bar{\zeta}_{i}=0
$$

In keeping with the discussion of the previous sections we will mostly focus on the holomorphic sector. The positions $\sigma_{i}^{a}$ and $\zeta_{i}^{a}$ are regarded as new constant fields which we integrate over in the path integral. This is somewhat unconventional but as demonstrated in [20] for the case of the bosonic string it allows for a uniform derivation of scattering amplitudes with integrated and unintegrated vertex operators. Here we extend that discussion to include the fermionic coordinates $\zeta_{i}^{a}$. The action (4.1) is invariant under diffeomorphisms provided one transforms the position $\sigma_{i}^{a}$ of the vertex operator $V_{i}$ and of $\zeta_{i}^{a}$ appropriately (the corresponding BRST transformations 
are given in (5.3) $)$. Furthermore, we need to ensure that (4.1) is $Q_{S}$ invariant. Since $\zeta_{i}$ is a fermionic variable $V_{i}$ has the expansion (in complex basis)

$$
V_{i}[\varphi]\left(z_{i}, \zeta_{i}\right)=V_{i}^{(0)}[\varphi]\left(z_{i}\right)+\zeta_{i} V_{i}^{(1)}[\varphi]\left(z_{i}\right)
$$

where again we focus on the holomorphic sector. For (4.1) to be $Q_{S}$ invariant, we need

$$
\delta_{S}\left(V_{i}[\varphi]\left(z_{i}, \zeta_{i}\right)\right)=0
$$

The $Q_{S}$ transformation can act either on worldsheet fields $\varphi$ or on the positions $z_{i}$ and we obtain

$$
\delta_{S} V_{i}[\varphi]\left(z_{i}, \zeta_{i}\right)=\left(\delta_{S} V_{i}^{(0)}\right)\left(z_{i}\right)+\zeta_{i}\left(\partial V_{i}^{(0)}\left(z_{i}\right)-\left(\delta_{S} V_{i}^{(1)}\right)\left(z_{i}\right)\right)
$$

which implies

$$
\delta_{S} V_{i}^{(0)}=0, \quad \delta_{S} V_{i}^{(1)}=\partial V_{i}^{(0)},
$$

where now $Q_{S}$ acts only on the fields. ¿From (4.7) we find that the integrated vertex operator

$$
U_{i}=\int d z V_{i}^{(1)}
$$

is $Q_{S}$ invariant.

\section{BRST quantization}

The action (4.1) constructed in the previous section is invariant under diffeomorphisms and local Weyl transformations. We will now proceed to quantize this system using standard BRST methods. As in [20, 25], our BRST analysis includes the "gauge invariances" due to zero modes. This is done using the Batalin-Vilkovisky (BV) quantization scheme [26, 27]. Our treatment is a straightforward extension of the analysis in [20], so we will mostly quote results; for a detailed discussion of the method including a concise self-contained summary of BV we refer to [20].

We introduce diffeomorphism and Weyl ghosts, $c^{a}$ and $C_{\omega}$, and their $Q_{S}$ partners,

$$
\delta_{S} c^{a}=\gamma^{a}, \quad \delta_{S} C_{\omega}=\gamma_{\omega}
$$

The BRST transformations of all fields are given as usual by replacing the gauge parameter by the corresponding ghost. We will need below the explicit transformation 
of the metric and its $Q_{S}$ partner,

$$
\delta_{V} g_{a b}=\mathcal{L}_{c} g_{a b}+2 C_{\omega} g_{a b}, \quad \delta_{V} \hat{\psi}_{a b}=\mathcal{L}_{c} \hat{\psi}_{a b}-\mathcal{L}_{\gamma} g_{a b}-2 \gamma_{\omega} g_{a b}+2 C_{\omega} \hat{\psi}_{a b}
$$

and of the positions of the vertex operators and their $Q_{S}$ partners,

$$
\delta_{V} \sigma_{i}^{a}=-c^{a}\left(\sigma_{i}\right), \quad \delta_{V} \zeta_{i}^{a}=\gamma^{a}\left(\sigma_{i}\right)
$$

As discussed in [20], the zero modes of the ghost fields are associated with a gauge invariance of the ghost action which should be gauge fixed. Following the BV quantization scheme, one should introduce ghost-for-ghosts, extraghosts, antighosts and associated auxiliary fields to gauge fix this invariance. For the problem at hand, (as explained in [20]) all these "fields" are constant, i.e. do not depend on the worldsheet coordinates, ghosts-for-ghosts are not needed and the metric moduli $\tau^{k}, k=$ $1, \ldots, 6 g-6,(g \geq 2)$, (considered as constant fields) play the role of extraghosts 3 . Recalling that due to the $Q_{S}$ symmetry all fields come in $Q_{S}$-multiplets, we end up introducing the following constant fields and BRST transformations,

$$
\delta_{S} \tau^{k}=\hat{\tau}^{k}, \quad \delta_{V} \tau^{k}=\xi^{k}, \quad \delta_{S} \xi^{k}=\hat{\xi}^{k}, \quad \delta_{V} \hat{\tau}^{k}=-\hat{\xi}^{k}
$$

We further need antighost fields and corresponding auxiliary fields

$$
\delta_{S} \tilde{\beta}^{a b}=\tilde{b}^{a b}, \quad \delta_{V} \tilde{\beta}^{a b}=-p^{a b}, \quad \delta_{V} \tilde{b}^{a b}=\pi^{a b}, \quad \delta_{S} p^{a b}=\pi^{a b}
$$

(which in our conventions are tensor densities).

To gauge fix diffeomorphism and Weyl transformations we set the worldsheet metric $g_{a b}$ equal to a reference metric $\hat{g}_{a b}(\tau)$. This can be implemented in the path integral via the following gauge fixing Lagrangian,

$$
\begin{aligned}
L_{1} & =\delta_{V} \delta_{S}\left(\tilde{\beta}^{a b}\left[g_{a b}-\hat{g}_{a b}(\tau)\right]\right) \\
& =\delta_{V}\left(\tilde{b}^{a b}\left[g_{a b}-\hat{g}_{a b}(\tau)\right]+\tilde{\beta}^{a b}\left[\hat{\psi}_{a b}-\hat{\tau}^{k} \partial_{k} \hat{g}_{a b}(\tau)\right]\right) \\
& =\pi^{a b}\left[g_{a b}-\hat{g}_{a b}(\tau)\right]-\tilde{b}^{a b}\left[2 C_{\omega} g_{a b}+\mathcal{L}_{c} g_{a b}-\xi^{k} \partial_{k} \hat{g}_{a b}(\tau)\right]-p^{a b}\left[\hat{\psi}_{a b}-\hat{\tau}^{k} \partial_{k} \hat{g}_{a b}(\tau)\right] \\
& +\tilde{\beta}^{a b}\left[\mathcal{L}_{c} \hat{\psi}_{a b}+2 C_{\omega} \hat{\psi}_{a b}-\mathcal{L}_{\gamma} g_{a b}-2 \gamma_{\omega} g_{a b}+\hat{\xi}^{k} \partial_{k} \hat{g}_{a b}(\tau)-\hat{\tau}^{k} \xi^{l} \partial_{k} \partial_{l} \hat{g}_{a b}(\tau)\right]
\end{aligned}
$$

${ }^{3}$ Earlier works where the moduli were treated as quantum mechanical degrees of freedom include [28, 29, 30, 25]. 
where $\partial_{k} \hat{g}_{a b}(\tau)=\partial \hat{g}_{a b}(\tau) / \partial \tau^{k}$ is the derivative of the reference metric w.r.t. the moduli and $\hat{\psi}_{a b}$ is defined in (3.14). This gauge fixing action contains the usual gauge fixing terms for the metric and the ghost actions for $\tilde{b}, c$ and $\tilde{\beta}, \gamma$.

In addition when the Riemann surface has $\kappa$ conformal Killing vectors 4 we need to fix $\kappa$ additional constant "gauge" symmetries. This is done by fixing the position of $\kappa$ vertex operators; we call this set $f$. From now on $\sigma_{i}^{a}$ will always belong to the complement of $f$ and $\sigma_{\hat{i}}^{a}$ to $f$. We further introduce additional constant antighosts and associated auxiliary fields,

$$
\delta_{S} \beta_{a}^{\hat{j}}=b_{a}^{\hat{j}},, \quad \delta_{V} \beta_{a}^{\hat{j}}=-p_{a}^{\hat{j}}, \quad \delta_{V} b_{a}^{\hat{j}}=\pi_{a}^{\hat{j}}, \quad \delta_{S} p_{a}^{\hat{j}}=\pi_{a}^{\hat{j}} \quad(a, \hat{j}) \in f
$$

and the following gauge fixing term

$$
\begin{aligned}
L_{2} & =\delta_{V} \delta_{S}\left(\sum_{f} \beta_{a}^{\hat{j}}\left(\sigma_{\hat{j}}^{a}-\hat{\sigma}_{\hat{j}}^{a}\right)\right)=\delta_{V}\left(\sum_{f} b_{a}^{\hat{j}}\left(\sigma_{\hat{j}}^{a}-\hat{\sigma}_{\hat{j}}^{a}\right)+\beta_{a}^{\hat{j}} \zeta_{\hat{j}}^{a}\right) \\
& =\sum_{f} \pi_{a}^{\hat{j}}\left(\sigma_{\hat{j}}^{a}-\hat{\sigma}_{\hat{j}}^{a}\right)-b_{a}^{\hat{j}} c^{a}\left(\sigma_{\hat{j}}\right)-p_{a}^{\hat{j}} \zeta_{\hat{j}}^{a}+\beta_{a}^{\hat{j}} \gamma^{a}\left(\sigma_{\hat{j}}\right) .
\end{aligned}
$$

At this point we have treated all gauge symmetries, except the ones associated with zero modes of the original fields $X, p, \theta, w, \lambda$. We will discuss these in the next section.

To summarize, the generating functional of scattering amplitudes is given by

$$
Z\left[\sigma_{i} ; \rho^{i}\right]=\int d \mu_{\sigma} d \mu \exp \left(-\mathcal{S}-L_{1}-L_{2}\right)
$$

where $\mathcal{S}, L_{1}$ and $L_{2}$ are given in (4.1), (5.6) and (5.8), $d \mu_{\sigma}$ is the measure factor associated with $X, p, \theta, w, \lambda$ (and non-minimal variables) that we will discuss in the next section and $d \mu$ is the measure that follows from the analysis of this section, i.e.

$$
\begin{aligned}
d \mu & =\prod_{i}^{n} d^{2} \sigma_{i} \sqrt{g\left(\sigma_{i}\right)} d^{2} \zeta_{i} \prod_{k=1}^{6 g-6} d \tau^{k} d \xi^{k} d \hat{\tau}^{k} d \hat{\xi}^{k} \prod_{f} d b_{a}^{\hat{j}} d p_{a}^{\hat{j}} d \beta_{a}^{\hat{j}} d \pi_{a}^{\hat{j}} \times \\
& \times\left[d \psi_{a b}\right]\left[d g_{a b}\right]\left[d c^{a}\right]\left[d \gamma^{a}\right]\left[d C_{\omega}\right]\left[d \gamma_{\omega}\right]\left[d p^{a b}\right]\left[d \tilde{\beta}^{a b}\right]\left[d \pi^{a b}\right]\left[d \tilde{b}^{a b}\right]
\end{aligned}
$$

The first line contains the integration over all constant "fields" while the second line the fields we functionally integrate over. The integration over most of these variables can be done exactly as we now discuss.

\footnotetext{
${ }^{4}$ Recall that $\kappa=6$ for a Riemann surface of genus $0, \kappa=2$ for genus one and $\kappa=0$ for higher genus surfaces.
} 
As in previous sections we only discuss the holomorphic sector. Firstly, integrating over $\pi^{a b}$ and $g_{a b}$ sets the worldsheet metric equal to the reference metric $\hat{g}_{a b}$ in all expressions. Integrating over $\pi_{a}^{\hat{j}}, p_{a}^{\hat{j}}$, leads to delta functions $\delta\left(z_{\hat{j}}-\hat{z}_{\hat{j}}\right) \delta\left(\zeta_{\hat{j}}\right)$ which can be used to integrate over $z_{\hat{j}}, \zeta_{\hat{j}}$. So $\kappa$ insertions 5 will involve $V_{\hat{j}}^{(0)}\left(\hat{z}_{\hat{j}}\right)$ while the remaining $(n-\kappa)$ vertex operators will involve $V_{i}^{(1)}\left(z_{i}\right)$ and will be integrated. Furthermore integrating out $b^{\hat{j}}, \beta^{\hat{j}}$ leads to the insertion $c\left(\hat{z}_{\hat{j}}\right) \delta\left(\gamma\left(\hat{z}_{\hat{j}}\right)\right)$.

Note that the $V_{\hat{j}}^{(0)}$ and $V_{i}^{(1)}$ do not depend on the ghost fields, so the path integral factorizes into a part that only depends on the ghosts and the rest. One might anticipate that the ghost contributions will cancel each other since $c^{a}, C_{\omega}$ and the $\gamma^{a}, \gamma_{\omega}$ are related by the $Q_{S}$ symmetry. So to simplify the presentation we set to zero the ghosts. The complete computation including the ghosts is given in appendix B, The scattering amplitudes thus take the form

$$
\left\langle V_{1} \cdots V_{n}\right\rangle=\int d \mu_{\sigma} e^{-S_{\sigma}} d \tilde{\mu} e^{-\tilde{S}} \prod_{\hat{j}=1}^{\kappa} V_{\hat{j}}^{(0)}\left(\hat{z}_{\hat{j}}\right) \prod_{i=\kappa+1}^{n} \int d z_{i} V_{i}^{(1)}\left(z_{i}\right),
$$

where

$$
d \tilde{\mu} e^{-\tilde{S}}=\prod_{k=1}^{6 g-6} d \tau^{k} d \hat{\tau}^{k}\left[d \psi_{a b}\right]\left[d p^{a b}\right] \exp \int d^{2} \sigma\left(\sqrt{\hat{g}} \frac{1}{2} G^{a b} \hat{\psi}_{a b}+p^{a b}\left[\hat{\psi}_{a b}-\hat{\tau}^{k} \partial_{k} \hat{g}_{a b}(\tau)\right]\right)
$$

Integrating out $p^{a b}$ gives a delta function that sets $\hat{\psi}_{a b}=\hat{\tau}^{k} \partial_{k} \hat{g}_{a b}(\tau)$. Finally integrating out $\hat{\tau}^{k}$ leads to $(6 g-6)$ (of which $(3 g-3)$ are holomorphic) insertions of $G^{a b}$,

$$
\left\langle V_{1} \cdots V_{n}\right\rangle=\int d \mu_{\sigma} e^{-S_{\sigma}} \prod_{k} d \tau^{k}\left(G, \partial_{k} \hat{g}\right) \prod_{\hat{j}=1}^{\kappa} V_{\hat{j}}^{(0)}\left(\hat{z}_{\hat{j}}\right) \prod_{i=\kappa+1}^{n} \int d z_{i} V_{i}^{(1)}\left(z_{i}\right)
$$

where $\left(G, \partial_{k} \hat{g}\right)=\int_{\Sigma} d^{2} \sigma \sqrt{\hat{g}} G^{a b} \partial_{k} \hat{g}_{a b}$.

\subsection{Summary}

Let us summarize the results so far. We started from a theory with a fermionic nilpotent symmetry $Q_{S}$ and zero central charge and we coupled it to topological gravity in a way that preserves the $Q_{S}$ symmetry. Quantizing this system using standard

\footnotetext{
${ }^{5}$ In the holomorphic section $\kappa=3$ for a Riemann surface of genus $0, \kappa=1$ for genus one and $\kappa=0$ for higher genus surfaces.
} 
BRST-BV methods leads to the formula (5.13) for the scattering amplitudes. In this formula the position of $\kappa$ of the vertex operators $V_{i}^{(0)}$ is fixed while the remaining ones, $V_{i}^{(1)}$, are integrated. These vertex operators satisfy (in the holomorphic sector),

$$
\delta_{S} V_{i}^{(0)}=0, \quad \delta_{S} V_{i}^{(1)}=\partial V_{i}^{(0)}
$$

Furthermore, one needs $(6 g-6)$ insertions $((3 g-3)$ holomorphic ones $)$ of the field $G_{a b}$ defined by

$$
\delta_{S} G_{a b}=T_{a b}
$$

where $T_{a b}$ is the stress energy tensor of the worldsheet theory. This composite field is the analogue of the $b$-antighost in the scattering prescription of bosonic string theory. One may have anticipated these results based on the scattering amplitude prescription for the bosonic string and studies of topological strings. Indeed this is precisely the prescription used in the literature. The novelty here is its derivation from a first principles BRST-BV quantization. Notice that these results hold irrespectively of what the original sigma model is. In the next section we discuss issues specific to the pure spinor theory described in section 2.

\section{Pure spinor measure}

We now return to the pure spinor sigma model. We would like to understand the path integral measure $d \mu_{\sigma}$ and find the explicit form of $G_{a b}$. The path integral measure will be derived by gauge fixing "invariances due to zero modes", as in the previous section. There is an important difference however. The vertex operators in general depend on all fields $X, \theta, \pi, w, \lambda$, so the zero modes imply only an invariance of the action $S_{\sigma}$ in (2.1) and not of the generating functional of correlators in (5.9). At first sight it seems as though one need not gauge fix this invariance of $S_{\sigma}$. Indeed fermionic zero modes do not present a problem; the vertex operators can provide the appropriate number of fermionic zero modes so that the final expressions are nonvanishing. Non-compact bosonic zero modes however are still a problem, even in the presence of vertex operators, because typically integration over them leads to a divergent path integral; the action $S_{\sigma}$ does not contain a convergence factor because

of the zero mode gauge invariance. This can be remedied by gauge fixing the bosonic 
zero mode gauge invariances, as we discuss in this section. As we shall see, because of the $Q_{S}$ invariance, part of the invariance due to fermionic zero modes is also fixed.

On a genus $g$ surface, a worldsheet scalar $\Phi$ has one zero mode $\Phi_{0}$ and a worldsheet vector $P$ has $g$ zero modes, $P_{0}(z)=\sum_{I=1}^{g} P^{I} \omega_{I}(z)$, where $\omega_{I}(z)$ are the $g$ holomorphic Abelian differentials of first kind satisfying $\int_{A_{I}} d z \omega_{J}=\delta_{I J}$ and the contour integral is around the $g$ non-trivial A-cycles of a genus $g$ surface. Note that $\Phi_{0}$ and $P^{I}$ are constants. In our case and in the minimal formulation we have 10 zero modes $x_{0}^{m}, 16$ zero modes $\theta_{0}^{\alpha}$ and 11 zero modes $\lambda_{0}^{\alpha}$ from the worldsheet scalars and $16 \mathrm{~g}$ zero modes $d_{\alpha}^{I}, I=1, \ldots g$, and $11 g$ zero modes $w_{\alpha}^{I}$ from the worldsheet vectors. Of these $x_{0}^{m}, \lambda_{0}^{\alpha}$ and $w_{\alpha}^{I}$ are bosonic. The treatment of the zero modes of $x^{m}$ is standard and will not be discussed here. Furthermore, following earlier work we will trade $w_{\alpha}$, which transforms under the gauge transformation (2.3), for the gauge invariant variables,

$$
N_{m n}=\frac{1}{2} w_{\alpha}\left(\gamma_{m n}\right)^{\alpha}{ }_{\beta} \lambda^{\beta}, \quad J=w_{\alpha} \lambda^{\alpha}
$$

where $N_{m n}$ is the (contribution of the pure spinors to the) Lorentz current and $J$ is the ghost generator. As discussed in [6], the pure spinor condition implies enough relations between $N_{m n}$ and $J$ so that one can express the 11 independent components of $w_{\alpha}$ in terms of $J$ and 10 component of $N_{m n}$. In what follows the $11 g$ zero modes of $N_{m n}, J$ will be denoted by $N_{m n}^{I}, J^{I}$.

The BRST transformations corresponding to the zero mode gauge invariance are given by

$$
\delta_{V} \lambda_{0}^{\alpha}=c^{\alpha}, \quad \delta_{V} \theta_{0}^{\alpha}=\gamma^{\alpha}, \quad \delta_{V} d_{\alpha}^{I}=\gamma_{\alpha}^{I}, \quad \delta_{V} w_{\alpha}^{I}=c_{\alpha}^{I},
$$

where $c^{\alpha}, c_{\alpha}^{I}$ are constant fermionic ghosts and $\gamma^{\alpha}, \gamma_{\alpha}^{I}$ are constant bosonic ghosts. The transformations for $\lambda_{0}^{\alpha}, w_{\alpha}^{I}$ require some explanation, since $\lambda^{\alpha}$ satisfy a quadratic constraint and $w_{\alpha}$ has a gauge invariance. These zero modes are most easily described in $U(5)$ variables since the system in terms of $\lambda^{+}, \lambda^{a b}, w_{+}, w_{a b}$ is unconstrained and has no gauge invariance (see appendix A). The BRST transformation is then given by shifting these variables by their zero modes. Reversing the steps in appendix A one may express $c^{\alpha}$ in terms of the 11 zero modes of $\lambda^{+}, \lambda^{a b}$ and $c_{\alpha}^{I}$ in terms of the $11 \mathrm{~g}$ zero modes of $w_{+}, w_{a b}$. The arbitrariness due to the gauge invariance (2.3) is then eliminated by passing to the gauge invariant variables $N_{m n}^{I}, J^{I}$. 
To maintain $Q_{S}$ invariance we further require

$$
\delta_{S} \gamma^{\alpha}=c^{\alpha}, \quad \delta_{S} c_{\alpha}^{I}=\gamma_{\alpha}^{I}
$$

To gauge fix the bosonic invariances we introduce constant fermionic and bosonic antighost fields, $b_{\alpha}, \tilde{b}_{\alpha}$ each containing 11 independent components, $\tilde{b}^{m n I}, b^{m n I}$, each containing $10 g$ independent components and $\tilde{b}^{I}, b^{I}$, each containing $g$ components and corresponding auxiliary fields. The $Q_{V}$ and $Q_{S}$ transformations of these fields are given by

$$
\begin{array}{lcc}
\delta_{S} b_{\alpha}=\tilde{b}_{\alpha}, & \delta_{S} b^{m n I}=\tilde{b}^{m n I}, & \delta_{S} b^{I}=\tilde{b}^{I} \\
\delta_{V} b_{\alpha}=-\pi_{\alpha}, & \delta_{V} \tilde{b}_{\alpha}=\tilde{\pi}_{\alpha} \quad & \delta_{V} b^{m n I}=-\pi^{m n I}, \\
\delta_{V} \tilde{b}^{m n I}=\tilde{\pi}^{m n I}, & \delta_{V} b^{I}=-\pi^{I}, & \delta_{V} \tilde{b}^{I}=\tilde{\pi}^{I} \\
\delta_{S} \pi_{\alpha}=\tilde{\pi}_{\alpha}, & \delta_{S} \pi^{m n I}=\tilde{\pi}^{m n I}, & \delta_{S} \pi^{I}=\tilde{\pi}^{I}
\end{array}
$$

To gauge fix the zero mode gauge invariances we now introduce the following gauge fixing Lagrangian

$$
\begin{aligned}
L_{3}= & \delta_{V} \delta_{S}\left(b_{\alpha} \theta_{0}^{\alpha}+\sum_{I=1}^{g}\left(b^{m n I} N_{m n}^{I}+b^{I} J^{I}\right)\right) \\
= & \delta_{V}\left(-b_{\alpha} \lambda_{0}^{\alpha}+\tilde{b}_{\alpha} \theta_{0}^{\alpha}+\sum_{I=1}^{g}\left(\frac{1}{2} b^{m n I}\left(d^{I} \gamma_{m n} \lambda_{0}\right)+\tilde{b}^{m n I} N_{m n}^{I}+b^{I}\left(d^{I} \lambda_{0}\right)+\tilde{b}^{I}\left(w^{I} \lambda_{0}\right)\right)\right) \\
= & \pi_{\alpha} \lambda_{0}^{\alpha}+\tilde{\pi}_{\alpha} \theta_{0}^{\alpha}+\sum_{I=1}^{g}\left(-\pi^{m n I} \frac{1}{2} d^{I} \gamma_{m n} \lambda_{0}+\tilde{\pi}^{m n I} N_{m n}^{I}-\pi^{I} d_{\alpha}^{I} \lambda_{0}^{\alpha}+\tilde{\pi}^{I} J^{I}\right) \\
& +b_{\alpha} c^{\alpha}+\tilde{b}_{\alpha} \gamma^{\alpha}+\sum_{I=1}^{g}\left(\frac{1}{2} b^{m n I}\left(\gamma^{I} \gamma_{m n} \lambda_{0}-d^{I} \gamma_{m n} c\right)-\frac{1}{2} \tilde{b}^{m n I}\left(c^{I} \gamma_{m n} \lambda_{0}-w^{I} \gamma_{m n} c\right)\right. \\
& \left.+b^{I}\left(\gamma^{I} \lambda_{0}-d^{I} c\right)-\tilde{b}^{I}\left(c^{I} \lambda_{0}-w^{I} c\right)\right)
\end{aligned}
$$

Integrating over $b^{\alpha}$ and $\tilde{b}^{\alpha}$ leads to delta functions for $c^{\alpha}$ and $\gamma^{\alpha}$, which can be used to integrate out $c^{\alpha}, \gamma^{\alpha}$. Integrating over $b^{m n I}, b^{I}, \tilde{b}^{m n I}, \tilde{b}^{I}$ yields $11 g$ delta functions $\delta\left(\gamma^{I} \gamma_{m n} \lambda_{0}\right) \delta\left(\gamma^{I} \lambda_{0}\right)\left(c^{I} \gamma_{m n} \lambda_{0}\right)\left(c^{I} \lambda_{0}\right)$. The same argument that implies that one can trade the $11 g$ zero modes of $w_{\alpha}$ for $N_{m n}^{I}$ and $J^{I}$ also implies that the delta functions set to zero $c^{I}, \gamma^{I}$ (with Jacobians canceling between the $\gamma^{I}$ and $c^{I}$ terms). So the 
zero-mode measure now becomes

$$
\begin{aligned}
& {\left[d \mu_{\sigma}\right]_{z . m .}=\left[d^{16} \theta_{0}\right]\left[d^{11} \tilde{\pi}\right]\left[d^{11} \lambda_{0}\right]\left[d^{11} \pi\right] \prod_{I=1}^{g}\left[d^{16} d^{I}\right]\left[d^{11} \pi_{I}\right]\left[d^{11} \tilde{\pi}_{I}\right]\left[d^{11} N_{I}\right] \times} \\
& \quad \times \exp \left(\pi_{\alpha} \lambda_{0}^{\alpha}+\tilde{\pi}_{\alpha} \theta_{0}^{\alpha}+\sum_{I=1}^{g}\left(-\pi^{m n I} \frac{1}{2} d^{I} \gamma_{m n} \lambda_{0}+\tilde{\pi}^{m n I} N_{m n}^{I}-\pi^{I} d_{\alpha}^{I} \lambda_{0}^{\alpha}+\tilde{\pi}^{I} J^{I}\right)\right)
\end{aligned}
$$

where $\left[d^{11} \lambda_{0}\right]$ and $\prod_{I}\left[d^{11} N_{I}\right]$ are the Lorentz invariant integration measures derived in [4], whose explicit form we will not need. Our focus here is on the factors coming from integrating over $\pi, \tilde{\pi}, \pi^{I}, \tilde{\pi}^{I}$.

\subsection{Minimal formulation}

Recall that $\pi_{\alpha}$ and $\tilde{\pi}_{\alpha}$ have 11 independent components each. One way to parametrize them is to write

$$
\pi_{\alpha}=p_{i} C_{\alpha}^{i}, \quad \tilde{\pi}_{\alpha}=\tilde{p}_{i} C_{\alpha}^{i}, \quad i=1, \ldots, 11
$$

where $p_{i}, \tilde{p}_{i}$ and the independent components and $C_{i}^{\alpha}$ is a constant matrix of rank 11. Then $\left[d^{11} \pi\right]\left[d^{11} \tilde{\pi}\right]=\prod_{i} d p_{i} d \tilde{p}_{i}$ and integrating over $p^{i}$ yields $\prod_{i} \delta\left(C_{\alpha}^{i} \lambda_{0}^{\alpha}\right)$, while integrating over $\tilde{p}^{i}$ yields $\prod_{i} C_{\alpha}^{i} \theta_{0}^{\alpha}$. Putting it differently, one may have started with antighosts and auxiliary field $b^{i}, \tilde{b}^{i}, p^{i}, \tilde{p}^{i}$ and gauge fixing condition $C_{\alpha}^{i} \lambda_{0}^{\alpha}=0$, for the invariance due to the 11 zero modes of $\lambda^{\alpha}$ and gauge fixing condition $C_{\alpha}^{i} \theta_{0}^{\alpha}=0$ for the invariance due to 11 of the 16 zero modes of $\theta$. Note that the insertions can be combined into 11 insertions of the "picture-lowering" operator

$$
Y_{C}=C_{\alpha} \theta_{0}^{\alpha} \delta\left(C_{\alpha} \lambda_{0}^{\alpha}\right)
$$

Similarly, we parametrize the $10 \mathrm{~g}$ independent components of $\pi^{m n I}$ and $\tilde{\pi}^{m n I}$ as

$$
\pi^{m n I}=p^{j I} B_{j I}^{m n}, \quad \tilde{\pi}^{m n I}=\tilde{p}^{j I} B_{j I}^{m n}, \quad j=1, \ldots, 10
$$

where $p^{j I}, \tilde{p}^{j I}$ are the $10 \mathrm{~g}$ independent components and $B_{j I}^{m n}$ are constants. Integrating over $p_{j I}, \tilde{p}_{j I}$ and $\pi_{I}, \tilde{\pi}_{I}$ leads to the insertions

$$
\prod_{I=1}^{g}\left(\left(d_{\alpha}^{I} \lambda_{0}^{\alpha}\right) \delta\left(J^{I}\right) \prod_{j=1}^{10} \frac{1}{2} B_{I j}^{m n}\left(d^{I} \gamma_{m n} \lambda_{0}\right) \delta\left(B_{I j}^{m n} N_{m n}^{I}\right)\right)=\prod_{R=1}^{g} Z_{J}\left(z_{R}\right) \prod_{P=1}^{10 g} Z_{B_{P}}\left(w_{P}\right)
$$

where we reassembled the insertions in terms of the "picture-raising" operators

$$
Z_{B}=\frac{1}{2} B^{m n} d \gamma_{m n} \lambda \delta\left(B^{m n} N_{m n}\right), \quad Z_{J}=\left(\lambda^{\alpha} d_{\alpha}\right) \delta(J)
$$


inserted at positions $z_{R}, w_{P}$. Here we use the fact that the non-zero modes in the r.h.s. of (6.10) do not contribute in any correlator [4]. These insertions correspond to gauge fixing conditions $B_{i J}^{m n} N_{m n}^{I}=0, J^{I}=0$, for the gauge invariance due to the $11 g w_{\alpha}$ zero modes and $B_{I j}^{m n}\left(d^{I} \gamma_{m n} \lambda_{0}\right)=0, d_{\alpha}^{I} \lambda_{0}^{\alpha}=0$ for the gauge invariance due to $11 \mathrm{~g}$ of the $16 \mathrm{~g}$ zero modes of $d_{\alpha}$. Note that the constants $C_{\alpha}^{i}, B_{j I}^{m n}$ enter through a gauge fixing term, so by standard arguments correlation functions do not depend on them and their presence does not imply breaking of Lorentz invariance.

What is left is to discuss $G_{a b}$. As we shall see, we only need to recall well known facts from the literature. By definition, $G_{a b}$ should satisfy (now in complex coordinates and dropping the indices)

$$
\delta_{S} G=T, \quad T=\frac{1}{2} \Pi^{m} \Pi_{m}+d_{\alpha} \partial \theta^{\alpha}-w_{\alpha} \partial \lambda^{\alpha}
$$

Since $\delta_{S}$ is nilpotent, this equation defines a cohomology class $[G]$, i.e. solutions $G$ up to $\delta_{S}$ exact terms. A solution of (6.12) is given by [31]

$$
G_{0}=\frac{C_{\alpha} G^{\alpha}}{C_{\alpha} \lambda^{\alpha}}, \quad G^{\alpha}=\frac{1}{2} \Pi^{m}\left(\gamma_{m} d\right)^{\alpha}-\frac{1}{4} N_{m n}\left(\gamma^{m n} \partial \theta\right)^{\alpha}-\frac{1}{4} J \partial \theta^{\alpha}-\frac{1}{4} \partial^{2} \theta^{\alpha},
$$

for a constant spinor $C_{\alpha}$. This expression also appeared in [16] as a twisted worldsheet supersymmetry current. This solution is however not acceptable because had we allowed for operators with behavior $\left(C_{\alpha} \lambda^{\alpha}\right)^{-1}$ the $Q_{S^{-}}$cohomology would be trivial. Indeed, consider the field $\xi$

$$
\xi=\frac{C_{\alpha} \theta^{\alpha}}{C_{\alpha} \lambda^{\alpha}}, \quad \delta_{S} \xi=1
$$

Then any closed operator $V$ is also exact since

$$
\delta_{S} V=0 \quad \Rightarrow \quad V=\delta_{S}(\xi V)
$$

A related issue is that the positions of the poles of $G_{0}$ are also the positions of the zeros of the path integral insertions thus making the expressions ill-defined.

One might hope to arrive at a well-defined expression by finding a different representative of the cohomology class $[G]$ such that the poles in the new $G$ would cancel against zeros in other path integration insertions. Indeed, such a representative $G_{1}$ exists and it is given by $G_{1}=b_{B} / Z_{B}$, where $Z_{B}$ is the picture raising operator in (6.11) and $b_{B}$ is the "picture-raised b ghosts" constructed in [4] by solving the equation,

$$
\delta_{S} b_{B}=Z_{B} T
$$


It was shown in [32] that $G_{1}$ is in the same cohomology class as $G_{0}$. Using this solution we find that the poles of $G_{1}$ indeed cancel against zeros coming from the picture raising operators.

Combining all ingredients we find that the multi-loop amplitude should include $3 g-3$ insertions of $b_{B}, 10 g-(3 g-3)$ insertions of $Z_{B}, g$ insertions of $Z_{J}$ and 11 insertions of $Y_{C}$. This is precisely the prescription proposed in [6].

\subsection{Non-minimal formulation}

Let us now return back to (6.6) and recall that $\pi_{\alpha}$ and $\tilde{\pi}_{\alpha}$ are $Q_{S}$ partners, $\delta_{S} \pi_{\alpha}=\tilde{\pi}_{\alpha}$, see (6.4), and each has 11 independent components. These are precisely the properties of the non-minimal variables $\bar{\lambda}_{\alpha}$ and $r_{\alpha}$, see section 2 , so one may identify

$$
\pi_{\alpha}=\bar{\lambda}_{\alpha}^{0}, \quad \tilde{\pi}_{\alpha}=r_{\alpha}^{0}
$$

where $\bar{\lambda}_{\alpha}^{0}, r_{\alpha}^{0}$ are the zero modes of $\bar{\lambda}_{\alpha}$ and $r_{\alpha}$. Actually since the non-minimal variables are cohomologically trivial their non-zero modes do not contribute to any observable and one may only keep their zero modes. Recall also that the non-minimal sector has a gauge invariance similar to (2.3) (whose explicit form is not needed here) and the following combinations are gauge invariant [5]

$$
\begin{aligned}
& \bar{N}_{m n}=\frac{1}{2}\left(\bar{w} \gamma_{m n} \bar{\lambda}-s \gamma_{m n} r\right), \quad \bar{J}=\bar{w}^{\alpha} \bar{\lambda}_{\alpha}-s^{\alpha} r_{\alpha}, \\
& S_{m n}=\frac{1}{2} s \gamma_{m n} \bar{\lambda}, \quad S=s^{\alpha} \bar{\lambda}_{\alpha}
\end{aligned}
$$

The canonical momenta $\bar{w}^{\alpha}$ and $s^{\alpha}$ have $11 g$ zero modes each which, as in the discussion of the minimal variables, can be traded for $10 \mathrm{~g}$ zero modes of $\bar{N}_{m n}^{I}$ and $S_{m n}^{I}$ and $g$ zero modes of $\bar{J}^{I}$ and $S^{I}$. Using the $Q_{S}$ transformations in (2.10) one finds

$$
\delta_{S} S_{m n}^{I}=\bar{N}_{m n}^{I}, \quad \delta_{S} S^{I}=\bar{J}^{I}
$$

We thus find that the fields $\bar{N}_{m n}^{I}, S_{m n}^{I}, S^{I}, \bar{J}^{I}$ have the same number of components and the same $Q_{S}$ transformations as $\pi^{m n I}, \tilde{\pi}^{m n I}, \pi^{I}, \tilde{\pi}^{I}$, and we can thus identify them,

$$
\pi^{m n I}=\bar{N}^{m n I}, \quad \tilde{\pi}^{m n I}=S_{m n}^{I}, \quad \pi^{I}=S^{I}, \quad \tilde{\pi}^{I}=\bar{J}^{I} .
$$

With these identifications the exponential factor in (6.6) is precisely the regularization factor $\mathcal{N}$ in [5] (up to inconsequential numerical factors). 
It remains to discuss $G_{a b}$. This field was constructed in [5] (with an elegant interpretation of the construction in terms of Čech cohomology given in [6])

$$
G_{B}=\frac{\bar{\lambda}_{\alpha} G^{\alpha}}{(\bar{\lambda} \lambda)}+\frac{\bar{\lambda}_{\alpha} r_{\beta} H^{[\alpha \beta]}}{(\bar{\lambda} \lambda)^{2}}-\frac{\bar{\lambda}_{\alpha} r_{\beta} r_{\gamma} K^{[\alpha \beta \gamma]}}{(\bar{\lambda} \lambda)^{3}}-\frac{\bar{\lambda}_{\alpha} r_{\beta} r_{\gamma} r_{\delta} L^{[\alpha \beta \gamma \delta]}}{(\bar{\lambda} \lambda)^{4}}
$$

where $G^{\alpha}$ is given in (6.13) and $H^{\alpha \beta}, K^{\alpha \beta \gamma}, L^{\alpha \beta \gamma \delta}$ are explicitly known but we will not need their detailed form here. Note also that this field is cohomologically equivalent to $G_{0}$ [33]. Combining all ingredients we thus arrive at the prescription proposed in [5].

Noticed that $G_{B}$ field has poles as $\bar{\lambda} \lambda \rightarrow 0$ so one might wonder whether this prescription suffers from the same problems as the one using $G_{0}$. Indeed, there is a non-minimal version of the argument around (6.14)-(6.15). The corresponding nonminimal $\xi$ field is [5]

$$
\xi_{n m}=\frac{\bar{\lambda}_{\alpha} \theta^{\alpha}}{\bar{\lambda}_{\beta} \lambda^{\beta}+r_{\beta} \theta^{\beta}}
$$

This diverges as $(\bar{\lambda} \lambda)^{-11}$ so one must ensure that no operators which diverge with this rate are allowed. A related issue is that the path integral with the insertions just discussed will diverge if the insertions diverge as fast as $(\bar{\lambda} \lambda)^{-11}$. As discussed in [5, 6] this can only happen for genus $g>2$ (since the pure spinor measure converges as $(\bar{\lambda} \lambda)^{11}$ and $G_{B}$ diverges as $\left.(\bar{\lambda} \lambda)^{-3}\right)$. One way to deal with this issue is look for a different representative $G_{(B, \epsilon)}$ of the $Q_{S}$ cohomology class of $[G]$ which is less singular than $G_{B}$ as $\bar{\lambda} \lambda \rightarrow 0$. A construction of such $G_{(B, \epsilon)}$ is presented in [6]. Using this $G_{(B, \epsilon)}$ field one then arrives at a prescription that in principle works to all orders.

This solves the problem in principle. The actual construction of $G_{(B, \epsilon)}$ however is very complicated. Given that the issues with singularities are related to the $\bar{\lambda} \lambda \rightarrow 0$ limit, a different approach would be to modify the gauge fixing condition for the pure spinor zero modes such that they are fixed to a non-zero value. It would be interesting to investigate if such gauge fixing can be implemented and whether it would lead to a simpler scattering amplitude prescription.

\section{Conclusions}

We presented in this paper a derivation of the scattering amplitude prescription of the pure spinor superstring from first principles. Our results confirmed the prescriptions 
advocated in [4] and [5, 6], show that these prescriptions are equivalent and also suggest avenues for searching for a simpler prescription.

We now summarize our approach. We considered the pure spinor model (i.e. the Green-Schwarz-Siegel action plus the pure spinor variables) as a "matter" sigma model with target space ten dimensional superspace (with embedding coordinates $X, \theta)$ times the pure spinor space (with embedding coordinates $\lambda$ ). To construct a string theory we coupled this model to two dimensional (topological) gravity and then quantized the resulting theory. One should contrast this approach with previous works where the aim was to find a model with local symmetry which upon gauge fixing would lead to the pure spinor model with $Q_{S}$ emerging as the BRST operator and the pure spinors $\lambda$ as the corresponding ghosts. For us $Q_{S}$ and $\lambda$ are part of the model $a b$ initio and the justification for starting with this model is that the $Q_{S}$ cohomology gives the superstring spectrum. To maintain the $Q_{S}$ symmetry and consistently quantize the model after coupling to $2 d$ gravity, the $Q_{S}$ symmetry had to be extended to act on the gravitational sector and we showed that $Q_{S}$ invariance requires the existence of a (composite) field $G$ whose $Q_{S}$ variation is equal to the $2 d$ stress energy tensor.

This model was then quantized using standard BRST techniques, introducing diffeomorphism ghosts, their $Q_{S}$ partners, associated auxiliary fields etc. It turns out that all variables one introduces in this process can be explicitly integrated out resulting in a prescription for the scattering amplitudes involving (as usual) a number of unintegrated and a number of integrated vertex operators and $(3 g-3)$ (complex) insertions of the zero modes of $G$. This result holds in general for any system with a nilpotent symmetry coupled to topological gravity.

Our analysis included a BRST treatment of the gauge invariances due to zero modes; the presence of a zero mode implies an invariance of the action under a shift of the field by the corresponding zero mode. To gauge fix these invariances we introduced constant ghosts, antighosts and corresponding auxiliary fields. In the presence of vertex operators some of these invariances are lifted. Nevertheless, one must still gauge fix all (non-compact) bosonic invariances because their presence implies that the worldsheet action does not provide the appropriate convergence factor for the integration over them. We carried out this analysis for the bosonic zero modes of 
the pure spinor sigma model. This led (among other things) to the introduction of constant auxiliary fields needed to implement the gauge fixing conditions in the path integral. Depending on the parametrization of these fields one is led either to the minimal [4] or the non-minimal [5] prescription for scattering amplitudes. In the latter case the auxiliary fields can be identified with the non-minimal variables (more precisely, the zero modes of the non-minimal variables, but since these variables are cohomologically trivial their non-zero modes do not contribute to any observable). To complete the construction one needs the explicit form of the composite " $b$-field" $G$. The relevant results in the literature nicely fit with our analysis and we thus arrived at the precise form of the scattering amplitude prescriptions in [4] and [5].

The most complicated part of the scattering amplitude prescription is the construction of a composite "b-field" with appropriate singular behavior. Although the existence of a completely satisfactory $G$ field is guaranteed by the results of [6], the actual construction is very complicated. A possible avenue towards a simpler prescription would be to look for different gauge fixing conditions for the zero modes, instead of looking for less singular representatives of $[G]$ as has been done so far. We hope to report on this and related issues in the future.

\section{Acknowledgments}

We would like to thank Nathan Berkovits and Carlos Mafra for discussions.

\section{A $\quad U(5)$ variables and the $Y$-formalism}

We discuss in this appendix the use of $U(5)$ variables and the $Y$-formalism. We start by relaxing the pure spinor condition on $\lambda^{\alpha}$ and introducing a Lagrange multiplier $l_{m}$ to impose it in the path integral. The $(w, \lambda)$ part of the action (2.1) thus now reads

$$
S_{(w, \lambda)}=\int d^{2} z\left(w_{\alpha} \bar{\partial} \lambda^{\alpha}+l_{m}\left(\lambda \gamma^{m} \lambda\right)\right)
$$

where $\lambda^{\alpha}$ is now an unconstrained chiral spinor. This action has a gauge invariance,

$$
\delta w_{\alpha}=\Lambda_{m}\left(\gamma^{m} \lambda\right)_{\alpha}, \quad \delta l_{m}=\frac{1}{2} \bar{\partial} \Lambda_{m}+\left(\Lambda \gamma_{m} \lambda\right)
$$


where $\Lambda^{m}$ and $\Lambda^{\alpha}$ are gauge parameters. The $\Lambda^{\alpha}$ gauge invariance follows from the Fierz identity

$$
\left(\lambda \gamma^{m} \lambda\right) \gamma_{m} \lambda=0
$$

that holds for any spinor $\lambda$. The same identity also implies that the gauge algebra is reducible; the gauge transformations are invariant under the transformation

$$
\delta \Lambda^{\alpha}=\left(\left(\lambda \gamma^{n} \lambda\right) \gamma_{n}^{\alpha \beta}-2 \lambda^{\alpha} \lambda^{\beta}\right) \tilde{\Lambda}_{\beta}
$$

with $\tilde{\Lambda}_{\beta}$ a new gauge parameter. This transformation has a gauge invariance of its own, etc. The full set of reducibility conditions is discussed in the appendix of [34] and in [35]. One may proceed to quantize this system in a manifestly Lorentz invariant fashion by introducing ghosts-for-ghosts etc but we shall not discuss this here. Instead we will use a Lorentz breaking gauge fixing condition.

Let $\Gamma^{m}$ be the $S O(10)$ gamma matrices and let us define

$$
\Gamma_{a}^{+}=\frac{1}{2}\left(\Gamma^{2 a}+i \Gamma^{2 a-1}\right), \quad \Gamma^{-a}=\frac{1}{2}\left(\Gamma^{2 a}-i \Gamma^{2 a-1}\right), \quad a=1,2,3,4,5 .
$$

The spinor representation can be built by treating $\Gamma^{a-}$ as annihilation and $\Gamma_{a}^{+}$as creation operators, where $a=1, \ldots, 5$. Let us define

$$
v_{a_{1} \ldots a_{n}}=\Gamma_{a_{1}}^{+} \ldots \Gamma_{a_{n}}^{+}|0\rangle, \quad v_{+}=\Gamma_{1}^{+} \Gamma_{2}^{+} \Gamma_{3}^{+} \Gamma_{4}^{+} \Gamma_{5}^{+}|0\rangle \quad v_{-}=|0\rangle
$$

where $n=1, \ldots, 4$. A chiral spinor has components $\lambda^{+}, \lambda^{a b}, \lambda_{a}=\epsilon_{a b c d e} \lambda^{b c d e} / 24$, which transform as $\underline{1}, \underline{10}$ and $\underline{5}^{*}$ under the $U(5)$ subgroup of $S O(10)$. In these variables only 5 of the 10 expressions $\lambda \gamma^{m} \lambda$ are non-trivial; the other 5 are automatically equal to zero if the first 5 hold,

$$
\begin{aligned}
\lambda \gamma_{a}^{+} \lambda & =2 \lambda_{a} \lambda^{+}+\frac{1}{4} \epsilon_{a b c d e} \lambda^{b c} \lambda^{d e} \\
\lambda \gamma^{a-} \lambda & =-2 \lambda_{b} \lambda^{a b}
\end{aligned}
$$

Using (A.7) one finds that (A.8) is automatically satisfied so without loss of generality we can set to zero the Lagrange multipliers $l_{a-}$. The action is now invariant under

$$
\delta w_{\alpha}=\Lambda^{a}\left(\gamma_{a}^{+} \lambda\right)_{\alpha}, \quad \delta l^{a+}=\frac{1}{2} \bar{\partial} \Lambda^{a}, \quad a=1, \ldots, 5
$$

This gauge transformation has rank 5, so one can gauge fix it by requiring

$$
w^{a}=0 .
$$


Following standard steps (and expressing the gamma matrices in the $U(5)$ basis) we find that corresponding ghost action is

$$
\int d^{2} z\left(\bar{C}_{b}\left(\gamma_{a}^{+}\right)^{b}{ }_{\beta} \lambda^{\beta} C^{a}+w^{a} \pi_{a}\right)=\int d^{2} z\left(\bar{C}_{a} \lambda^{+} C^{a}+w^{a} \pi_{a}\right)
$$

where $\bar{C}_{b}, C^{a}, \pi_{a}$ are the corresponding antighost, ghost and auxiliary fields. Integrating them out sets $w^{a}=0$ and inserts in the path integral measure the factor $\left(\lambda^{+}\right)^{5}$. Furthermore, integrating out $l^{a+}$ leads to the delta function $\delta\left(2 \lambda_{a} \lambda^{+}+\frac{1}{4} \epsilon_{a b c d e} \lambda^{b c} \lambda^{d e}\right)$ which can be used to integrate out $\lambda_{a}$ (so we are left with the 11 independent components $\left.\lambda^{+}, \lambda^{a b}\right)$ and also results in the insertion $\left(\lambda^{+}\right)^{-5}$ in the path integral measure, which cancel the factor $\left(\lambda^{+}\right)^{5}$ from the ghosts. The end result is that the action (A.1) becomes the free action

$$
\int d^{2} z\left(w_{+} \bar{\partial} \lambda^{+}+w_{a b} \bar{\partial} \lambda^{a b}\right)
$$

with all factors coming from eliminating the $\underline{5}^{*}$ and gauge fixing the gauge invariance canceling out.

¿From this local description one should now pass to the global picture by gluing together the local pieces. The general theory is presented in [21, 22] and the pure spinor case has been discussed in detail in [22]. In general, there may be worldsheet and target space diffeomorphism anomalies that render the theory inconsistent. These were shown to cancel in the pure spinor case if one would excise the $\lambda=0$ point from the space of pure spinors [22]. Furthermore, requiring consistent gluing should also fix the path integral measure. Since the theory is non-anomalous this measure should be the Lorentz invariant measure determined in [6] .

Finally, let us briefly discuss the $Y$-formalism of [36, 37, 33. In this case one introduces a constant pure spinor $v_{\alpha}$ and the following projector,

$$
K_{\alpha}^{\beta}=\frac{1}{2}\left(\gamma^{m} \lambda\right)_{\alpha}\left(Y \gamma_{m}\right)^{\beta}
$$

where $Y_{\alpha}=v_{\alpha} /\left(v_{\alpha} \lambda^{\alpha}\right)$. This projector has rank 5 (since $\operatorname{Tr} K=5$ ) and can be used

\footnotetext{
${ }^{6}$ To verify this one should first determine the measure in terms of $\lambda^{+}, \lambda^{a b}, w^{-}, w_{a b}$ requiring invariance of the measure when we move from one patch to another and then rewrite the resulting measure in a way that is manifestly Lorentz invariant. For the $w^{-}, w^{a b}$ variables this would involve changing variables to $N_{m n}, J$. As far as we are aware this computation has not appeared in the literature, see however [22].
} 
to solve the pure spinor condition,

$$
\left(\lambda \gamma^{m} \lambda\right)=0 \quad \Leftrightarrow \quad \lambda^{\alpha} K_{\alpha}^{\beta}=0
$$

Furthermore,

$$
(1-K)_{\alpha}^{\beta}\left(\gamma^{m} \lambda\right)_{\beta}=0,
$$

so $\left(\gamma^{m} \lambda\right)_{\beta}$ also has rank 5 . This means that the gauge invariance can eliminate 5 of the components of $w_{\alpha}$, which can be done using the gauge fixing condition,

$$
K_{\alpha}{ }^{\beta} w_{\beta}=0
$$

Following our earlier discussion, one should now implement these steps in the path integral. Up to issues related to possible path integral insertions that could result from the details of the integration over $l_{m}$ and the ghost, this should result in the $Y$ formalism.

\section{B Ghost contribution}

We discuss in this appendix the computation of the contribution of the ghost fields to scattering amplitudes. We will compute

$$
Z_{m}=\int d \tilde{\mu} e^{-\tilde{S}} d \mu_{g h} \exp \left(-S_{g h}\right)
$$

where $d \tilde{\mu} e^{-\tilde{S}}$ is given in $(\underline{5.12})$,

$$
d \mu_{g h}=\left[d \tilde{\beta}^{a b}\right]\left[d \tilde{b}^{a b}\right]\left[d c^{a}\right]\left[d \gamma^{a}\right]\left[d C_{\omega}\right]\left[d \gamma_{\omega}\right]\left[d \xi^{k}\right]\left[d \hat{\xi}^{k}\right] \prod_{\hat{j}=1}^{\kappa} c^{a}\left(\hat{\sigma}_{\hat{j}}\right) \delta\left(\gamma^{a}\left(\hat{\sigma}_{\hat{j}}\right)\right.
$$

and

$$
\begin{aligned}
S_{g h}= & \int_{\Sigma}\left(2 \gamma_{\omega} \tilde{\beta}^{a b} \hat{g}_{a b}(\tau)-2 C_{\omega}\left(\tilde{b}^{a b} \hat{g}_{a b}(\tau)-\tilde{\beta}^{a b} \hat{\psi}_{a b}\right)\right. \\
& +\tilde{b}^{a b}\left[\hat{\nabla}_{a} c_{b}+\hat{\nabla}_{b} c_{a}\right]+\tilde{\beta}^{a b}\left[\hat{\nabla}_{a} \gamma_{b}+\hat{\nabla}_{b} \gamma_{a}\right]+\tilde{b}^{a b} \xi^{k} \partial_{k} \hat{g}_{a b}(\tau) \\
& \left.-\hat{\psi}_{a b}\left[\partial_{c}\left(\tilde{\beta}^{a b} c^{c}\right)-2 \tilde{\beta}^{c(b} \partial_{c} c^{a)}\right]-\tilde{\beta}^{a b}\left[\hat{\xi}^{k} \partial_{k} \hat{g}_{a b}(\tau)-\hat{\tau}^{k} \xi^{l} \partial_{k} \partial_{l} \hat{g}_{a b}(\tau)\right]\right)
\end{aligned}
$$

where $\hat{\nabla}_{a}$ is the covariant derivative associated with $\hat{g}_{a b}$.

Integrating out $\gamma_{\omega}$ and $\beta(\tau) \equiv \hat{g}_{a b}(\tau) \tilde{\beta}^{a b}$ sets the trace of $\tilde{\beta}^{a b}$ equal to zero. We will denote by $\beta^{a b}$ the traceless part of $\tilde{\beta}^{a b}$. Integrating out $\hat{\xi}^{k}$ introduces $(6 g-6)$ insertions 
of the $\beta^{a b}$ zero modes, while integrating over $p^{a b}, \psi_{a b}$ and $\hat{\tau}^{k}$ leads to insertions of the zero mode of the "supercurrent",

$$
\left(\tilde{G}, \partial_{k} \hat{g}\right) \equiv \int_{\Sigma} d^{2} \sigma\left(\partial_{c}\left(\beta^{a b} c^{c}\right)-2 \beta^{c(b} \partial_{c} c^{a)}+2 \beta^{a b} C_{\omega}+\sqrt{\hat{g}} G^{a b}+\beta^{a b} \xi^{l} \partial_{l}\right) \partial_{k} \hat{g}_{a b}(\tau) .
$$

After these integrations we are left with

$$
Z_{m}=\int d \mu_{\beta \gamma} d \tilde{\mu}_{g h} \exp \left(-\tilde{S}_{g h}\right)
$$

where

$$
\tilde{S}_{g h}=\int d^{2} \sigma\left(\beta^{a b}\left(\hat{\nabla}_{a} \gamma_{b}+\hat{\nabla}_{b} \gamma_{a}\right)+\tilde{b}^{a b}\left(2 C_{\omega} \hat{g}_{a b}+\hat{\nabla}_{a} c_{b}+\hat{\nabla}_{b} c_{a}\right)+\tilde{b}^{a b} \xi^{k} \partial_{k} \hat{g}_{a b}(\tau)\right)
$$

and

$$
\begin{aligned}
& d \mu_{\beta \gamma}=\left[d \beta^{a b}\right]\left[d \gamma^{a}\right] \prod_{k=1}^{6 g-6} \delta\left(\left(\beta, \partial_{k} \hat{g}\right)\right) \prod_{\hat{j}=1}^{\kappa} \delta\left(\gamma^{a}\left(\hat{\sigma}_{\hat{j}}\right)\right) \\
& d \tilde{\mu}_{g h}=\left[d \tilde{b}^{a b}\right]\left[d c^{a}\right]\left[d C_{\omega}\right] \prod_{k=1}^{6 g-6} d \tau^{k} d \xi^{k}\left(\tilde{G}, \partial_{k} \hat{g}(\tau)\right) \prod_{\hat{j}=1}^{\kappa} c^{a}\left(\hat{\sigma}_{\hat{j}}\right)
\end{aligned}
$$

The $\beta \gamma$ system is now a standard CFT with a $U(1)$ "ghost" charge conservation and the path integral measure contains all appropriate zero mode insertions. It follows that the $\beta$-dependent part of $(\underline{B .4})$ drops out of $(\underline{B .5})$ since it is charged w.r.t. the $\beta \gamma \mathrm{U}(1)$. Integrating out $C_{\omega}$ sets the trace of $\tilde{b}^{a b}$ to zero; we will denote by $b^{a b}$ the traceless part, and integrating out $\xi^{k}$ leads to $(6 g-6)$ insertions of the $b^{a b}$ zero modes. We end up with

$$
Z_{m}=\int d \mu_{\tau} d \mu_{\beta \gamma} d \mu_{b c} \exp \left(-\int d^{2} \sigma\left(\beta^{a b}\left(\hat{\nabla}_{a} \gamma_{b}+\hat{\nabla}_{b} \gamma_{a}\right)+b^{a b}\left(\hat{\nabla}_{a} c_{b}+\hat{\nabla}_{b} c_{a}\right)\right)\right)
$$

with $d \mu_{\beta \gamma}$ as in (B.7) and

$$
\begin{aligned}
d \mu_{b c} & =\left[d b^{a b}\right]\left[d c^{a}\right] \prod_{k=1}^{6 g-6}\left(b, \partial_{k} \hat{g}(\tau)\right) \prod_{\hat{j}=1}^{\kappa} c^{a}\left(\hat{\sigma}_{\hat{j}}\right) \\
d \mu_{\tau} & =\prod_{k=1}^{6 g-6} d \tau^{k}\left(G, \partial_{k} \hat{g}(\tau)\right)
\end{aligned}
$$

It is now manifest that the integration over $\left(b^{a b}, c^{a}\right)$ cancels against the integration over $\left(\beta^{a b}, \gamma^{a}\right)$ and we are left with the same measure factor as in (5.13). 


\section{References}

[1] N. Berkovits, "Super-Poincare covariant quantization of the superstring," JHEP 0004, 018 (2000) arXiv:hep-th/0001035.

[2] N. Berkovits, "ICTP lectures on covariant quantization of the superstring," arXiv:hep-th/0209059.

[3] W. Siegel, "Classical Superstring Mechanics," Nucl. Phys. B 263, 93 (1986).

[4] N. Berkovits, "Multiloop amplitudes and vanishing theorems using the pure spinor formalism for the superstring," JHEP 0409, 047 (2004) arXiv:hep-th/0406055.

[5] N. Berkovits, "Pure spinor formalism as an $\mathrm{N}=2$ topological string," JHEP 0510, 089 (2005) arXiv:hep-th/0509120.

[6] N. Berkovits and N. Nekrasov, "Multiloop superstring amplitudes from non-minimal pure spinor formalism," JHEP 0612, 029 (2006) arXiv:hep-th/0609012.

[7] N. Berkovits and B. C. Vallilo, "Consistency of super-Poincare covariant superstring tree amplitudes," JHEP 0007, 015 (2000) arXiv:hep-th/0004171.

[8] N. Berkovits, "Super-Poincare covariant two-loop superstring amplitudes," JHEP 0601, 005 (2006) arXiv:hep-th/0503197].

[9] N. Berkovits and C. R. Mafra, "Equivalence of two-loop superstring amplitudes in the pure spinor and RNS formalisms," Phys. Rev. Lett. 96, 011602 (2006) arXiv:hep-th/0509234.

[10] G. Policastro and D. Tsimpis, "R**4, purified," Class. Quant. Grav. 23, 4753 (2006) arXiv:hep-th/0603165.

[11] N. Berkovits and C. R. Mafra, "Some superstring amplitude computations with the non-minimal pure spinor formalism," JHEP 0611, 079 (2006) arXiv:hep-th/0607187. 
[12] P. A. Grassi, G. Policastro, M. Porrati and P. Van Nieuwenhuizen, "Covariant quantization of superstrings without pure spinor constraints," JHEP 0210, 054 (2002) arXiv:hep-th/0112162.

[13] P. A. Grassi, G. Policastro and P. van Nieuwenhuizen, "An introduction to the covariant quantization of superstrings," Class. Quant. Grav. 20, S395 (2003) arXiv:hep-th/0302147.

[14] P. A. Grassi, G. Policastro and P. van Nieuwenhuizen, "The quantum superstring as a WZNW model," Nucl. Phys. B 676, 43 (2004) arXiv:hep-th/0307056.

[15] I. Oda and M. Tonin, "On the Berkovits covariant quantization of GS superstring," Phys. Lett. B 520, 398 (2001) arXiv:hep-th/0109051].

[16] M. Matone, L. Mazzucato, I. Oda, D. Sorokin and M. Tonin, "The superembedding origin of the Berkovits pure spinor covariant quantization of superstrings," Nucl. Phys. B 639, 182 (2002) arXiv:hep-th/0206104.

[17] Y. Aisaka and Y. Kazama, "Origin of pure spinor superstring," JHEP 0505, 046 (2005) arXiv:hep-th/0502208.

[18] N. Berkovits and D. Z. Marchioro, "Relating the Green-Schwarz and pure spinor formalisms for the superstring," JHEP 0501, 018 (2005) [arXiv:hep-th/0412198].

[19] A. Gaona and J. A. Garcia, "BFT embedding of the Green-Schwarz superstring and the pure spinor formalism," JHEP 0509, 083 (2005) arXiv:hep-th/0507076.

[20] B. Craps and K. Skenderis, "Comments on BRST quantization of strings," JHEP 0505, 001 (2005) arXiv:hep-th/0503038].

[21] E. Witten, "Two-dimensional models with $(0,2)$ supersymmetry: Perturbative aspects," arXiv:hep-th/0504078.

[22] N. A. Nekrasov, "Lectures on curved beta-gamma systems, pure spinors, and anomalies," arXiv:hep-th/0511008.

[23] N. Berkovits, "Cohomology in the pure spinor formalism for the superstring," JHEP 0009, 046 (2000) arXiv:hep-th/0006003. 
[24] R. Dijkgraaf, H. L. Verlinde and E. P. Verlinde, "Notes on topological string theory and 2-D quantum gravity," in Trieste Spring School 1990:0091-156.

[25] C. M. Becchi and C. Imbimbo, "Gribov horizon, contact terms and Čech- De Rham cohomology in 2D topological gravity," Nucl. Phys. B 462, 571 (1996) arXiv:hep-th/9510003].

[26] I. A. Batalin and G. A. Vilkovisky, "Quantization Of Gauge Theories With Linearly Dependent Generators," Phys. Rev. D 28, 2567 (1983) [Erratum-ibid. D 30, 508 (1984)].

[27] M. Henneaux and C. Teitelboim, "Quantization of gauge systems," Princeton, USA: University Press (1992).

[28] L. Baulieu and M. P. Bellon, "Brst Symmetry For Finite Dimensional Invariances: Applications To Global Zero Modes In String Theory," Phys. Lett. B 202, 67 (1988).

[29] J. M. F. Labastida, M. Pernici and E. Witten, "Topological Gravity in TwoDimensions," Nucl. Phys. B 310, 611 (1988).

[30] P. Mansfield, "The Consistency of topological expansions in field theory: 'BRST anomalies' in strings and Yang-Mills," Nucl. Phys. B 416, 205 (1994) arXiv:hep-th/9308117.

[31] N. Berkovits, "Relating the RNS and pure spinor formalisms for the superstring," JHEP 0108, 026 (2001) arXiv:hep-th/0104247.

[32] I. Oda and M. Tonin, "On the b-antighost in the pure spinor quantization of superstrings," Phys. Lett. B 606, 218 (2005) arXiv:hep-th/0409052.

[33] I. Oda and M. Tonin, "Y-formalism and $b$ ghost in the Non-minimal Pure Spinor Formalism of Superstrings," Nucl. Phys. B 779, 63 (2007) arXiv:0704.1219 [hepth]].

[34] N. Berkovits, "Covariant quantization of the superparticle using pure spinors," JHEP 0109, 016 (2001) arXiv:hep-th/0105050]. 
[35] N. Berkovits and N. Nekrasov, "The character of pure spinors," Lett. Math. Phys. 74, 75 (2005) arXiv:hep-th/0503075.

[36] I. Oda and M. Tonin, "Y-formalism in pure spinor quantization of superstrings," Nucl. Phys. B 727, 176 (2005) arXiv:hep-th/0505277.

[37] I. Oda and M. Tonin, "The b-field in pure spinor quantization of superstrings," arXiv:hep-th/0510223. 